\title{
9
}

\section{medicines information}

\section{ALLISON DONNELLY AND CAROL PATON}

\section{Safety of selective serotonin reuptake inhibitors in pregnancy}

\begin{abstract}
AIMS AND METHOD
Selective serotonin reuptake inhibitors (SSRIs) are recommended by the National Institute for Clinical Excellence as first-line drugs for the treatment of depression, but there is emerging evidence that they might not be entirely safe in pregnancy. We reviewed the literature in this area.
\end{abstract}

\author{
RESULTS \\ Some data indicate an association \\ between first-trimester SSRI expo- \\ sure, particularly with paroxetine, \\ and an increased risk of some major \\ congenital malformations. Stronger \\ evidence supports an association with \\ small reductions in gestational age \\ and neonatal withdrawal symptoms.
}

\author{
CLINICAL IMPLICATIONS \\ Risks and benefits of using SSRIs \\ during pregnancy should be dis- \\ cussed with the patient, and a joint \\ decision made between prescriber \\ and patient regarding treatment. \\ Limited data suggest that other SSRIs \\ are safer than paroxetine in \\ pregnancy.
}

Depression is common in women of child-bearing age with an annual prevalence of approximately 10\% (World Health Organization, 2001). Women who develop depression during pregnancy may be less likely to care for themselves, placing their own health and that of their unborn child at risk. For those who have an established diagnosis of depression and who are taking antidepressant drugs when they conceive, the risk of relapse on stopping their antidepressant is high. Cohen et al (2006) found that 44 of 65 women (68\%) who discontinued their antidepressant relapsed compared with 21 of 82 women $(26 \%)$ who continued their treatment. Women with a history of depression, particularly post-partum, are at a high risk of post-partum relapse. For those with bipolar disorder this may be up to 50\% (Cohen et al, 1995). These figures suggest that most psychiatrists who work with adults of working age are likely to be faced with making decisions about prescribing antidepressants for women who are pregnant or who wish to conceive.

The National Institute for Clinical Excellence (NICE) recommends selective serotonin reuptake inhibitors (SSRIs) as first-line treatment for moderate to severe depression (National Institute for Clinical Excellence, 2004). There have been a number of recent publications reporting on the safety of SSRIs in pregnancy and these studies are reviewed here.

\section{Background risk of congenital malformations}

The background risk of congenital malformations is 2-3\%; these figures are derived from a hospital-based surveillance study involving 69277 births (Nelson \&
Holmes, 1989). This background risk is known to be influenced by factors such as maternal age, genetics and the presence of some physical illnesses such as epilepsy. Exposure to drugs, particularly during the first trimester when the major organs are formed, can potentially further increase risk.

\section{Assessing the effects of drugs on pregnancy outcome}

It is obviously unethical to conduct randomised controlled trials in pregnant women as this would involve the intentional exposure of the foetus to a potential teratogen. Other methods commonly used to detect congenital malformations associated with drugs include pre-licensing studies in laboratory animals, and post-licensing observational studies in humans. Extrapolation of animal data to humans is complex and studies of this type will not be considered further here. Human data can originate from prospective or retrospective studies, all of which have limitations. Examples of prospective studies include cohort studies such as those conducted through teratology information services; women who contact these services may not be representative of pregnant women as a whole. Examples of retrospective studies are cohort and case-control studies; these types of study usually utilise administrative data from healthcare databases that have been set up for an entirely different purpose. Assumptions are made, such as every woman who is prescribed an antidepressant drug will actually take it. People cannot be directly questioned or physically examined. 
网

medicines information
The rate of congenital malformations in control groups identified from healthcare databases can vary widely depending on the demographic and clinical characteristics of the women selected as controls and the size of the group. It is important that the control group are matched for factors other than drug treatment that may affect the outcome that is being investigated. For example, if low birth weight is the outcome being measured, women should be matched for smoking status.

\section{Risks associated with SSRIs}

\section{Prospective cohort studies}

Einerson \& Einerson (2005) conducted a meta-analysis of studies from teratology information services. Women who contacted these services to ask for advice about the use of antidepressants in pregnancy were followed-up prospectively and pregnancy outcomes were compared with women who contacted the same information services requesting advice about drugs known not to be teratogenic. The overall malformation rate in the 830 women exposed to SSRIs and related antidepressants in the first trimester of pregnancy was $2.7 \%$ compared with $2.6 \%$ in women who were taking drugs that are known not to be teratogenic; no increase was seen in the malformation rate overall or in any specific malformation. This analysis was powered to detect specific malformations that occurred at a frequency of up to 1 in 240 exposures (i.e. were relatively common). The major limitations of this analysis were that:

- only live births were included; no information is available on the number of spontaneous abortions, elective abortions or still births

- women who contact teratology information services are likely to be more educated and from a higher social class than most women who are treated with drugs during pregnancy.

Since this meta-analysis was published, there has been a further prospective study from a teratology information service. Diav-Citrin et al (2005) followed up 330 paroxetine- and 230 fluoxetine-exposed pregnancies and compared outcomes with 1141 women who sought advice about exposure to drugs that are known not to be teratogenic. There was a higher rate of major abnormalities overall ( $R R=1.92,95 \% \mathrm{Cl} 1.01-3.65)$ and of cardiovascular abnormalities in particular $(\mathrm{RR}=3.46,95 \% \mathrm{Cl}$ $1.06-11.24)$ in infants exposed to paroxetine in the first trimester compared with controls. These calculations are based on 13 paroxetine-exposed infants with major congenital malformations and 5 with cardiac malformations, so should be treated with caution. Diav-Citrin et al (2005) also report that perinatal complications were more common in the SSRI-exposed group than the control group. This study is available in abstract form only and no further details are available.

In a further cohort study, Levinson-Castiel et al (2006) reported that almost a third of neonates exposed to SSRIs in the third trimester $(n=60)$ experienced symptoms that were attributed to SSRI withdrawal, such as sleep disturbance, tremor, hypertonicity, tachypnoea and gastrointestinal disturbance. None of the 60 unexposed neonates, matched for birth weight, gestation, Apgar score and mode of delivery, experienced these symptoms. Zeskind \& Stephens (2005) examined 17 infants exposed to SSRIs in utero and 17 non-exposed infants matched for maternal age, social class and smoking. Those infants who had been exposed to SSRIs showed disruption in a range of neurobehavioural outcomes such as tremulousness and sleep. The authors comment that it is unknown if these changes are enduring, and if they are, what the long-term consequences might be.

\section{Retrospective cohort studies}

Simon et al (2002) examined outcomes in 394 infants born to women who had been prescribed antidepressants during pregnancy and 394 infants whose mothers had not been prescribed antidepressants. All cases and controls were identified from the Group Health Cooperative database in Washington, USA (http:// www.ghc.org). No association between antidepressant exposure and foetal malformations, either overall or specific, was found, although it should be noted that the power of this study to detect differences was low. This negative finding has since been replicated in a much larger cohort of infants ( $n=1398)$ exposed to antidepressants in utero (Malm et al, 2005).

In a database study that is currently only available in abstract form, Wogelius et al (2005) reported that the odds ratio for congenital malformations in 1054 infants exposed to an SSRI in the first trimester was $1.4(95 \% \mathrm{Cl}$ 1.1-1.9) compared with infants not exposed to an SSRI in utero. The odds ratio for cardiac malformations in this study was 1.6 (95\% Cl 1.0-2.6). No further details are available and it is not clear if women were matched for the presence of depression.

The preliminary report of a database study that included 5956 women who were prescribed antidepressant drugs during the first trimester states that infants exposed to paroxetine $(n=815)$ were twice as likely to have a major congenital malformation as infants born to mothers who had been exposed to other antidepressants (GlaxoSmithKline, 2006); 1.5\% of infants exposed to paroxetine had cardiovascular malformations, compared with $1 \%$ exposed to other antidepressant drugs $(O R=1.5$, $95 \% \mathrm{Cl} 0.8-2.9)$. The majority were ventricular septal defects. Although this study is in line with the findings of Diav-Citrin et al (2005), the confidence intervals around the odds ratio cross unity, so some doubt about the certainty of the relationship remains.

Simon et al (2002) reported that infants born to mothers who had been prescribed SSRIs during pregnancy were more likely to have a lower gestational age: mean reduction 0.9 weeks with a fourfold increase in the risk of delivery before 36 weeks. Decreased gestational age was not noted in infants whose mothers had been prescribed tricyclic antidepressants during pregnancy. This 
association between SSRI use during pregnancy and decreased gestational age is consistent with that of several other studies including a Canadian cohort study (Wen et al, 2006) where $19.3 \%$ of 972 infants exposed to SSRIs were born before 37 weeks gestation v. $12 \%$ of 3878 unexposed infants ( $O R=1.57,95 \% \mathrm{Cl} 1.28-1.92)$; a Danish database study (Wogelius et al, 2005) where the number exposed to SSRIs was 994 (OR for birth prior to 37 weeks' gestation=1.4, 95\% Cl 1.1-1.8); and a Finnish database study (Malm et al, 2005) where the number exposed to SSRIs was 1782 (difference in mean gestational age was 0.3 weeks, $P<0.001$ ). A large Swedish birth registry study also replicated this finding (Kallen, 2004) (number exposed to SSRIs $=558$, OR for gestational age $<37$ weeks $=2.06,95 \% \mathrm{Cl} 1.58-2.69$ ) but failed to show a differential effect between exposure to SSRIs and exposure to tricyclic antidepressants. There are differences in methodology that might account for the spectrum of findings; the study of Simon et al (2002) was the only one to control for psychiatric history, whereas Malm et al (2005) did not control for alcohol use.

Simon et al (2002) reported that infants born to women who had been prescribed SSRIs in the third trimester had lower Apgar scores at birth, and Malm et al (2005) reported infants born to women who had taken SSRIs during the third trimester were more likely to require treatment in intensive care than infants who had not been exposed to SSRIs in utero (15.7 v. 11.2\%) despite no obvious difference in gestational age. These findings are consistent with the increased rate of perinatal complications in women exposed to SSRIs reported by Diav-Citrin et al (2005).

Sanz et al (2005) describe spontaneous reports of neonatal SSRI withdrawal syndrome to the World Health Organization database; in over two-thirds of cases, the neonate had been exposed to paroxetine in utero. Data up to November 2003 were included in this analysis, and 93 suspected cases were identified. Spontaneous reports are known to be influenced by many factors; published reports of the association between paroxetine and discontinuation symptoms in adults may have influenced reporting rates for this drug.

\section{Case-control studies}

Chambers et al (2006) conducted a case-control study of 377 women whose infants had persistent pulmonary hypertension of the newborn (PPHN), matched with 836 women whose infants did not have PPHN. This is a serious condition associated with a mortality rate of $10-20 \%$.

Those women who self-reported use of an SSRI after the 20th week of gestation were 6 times more likely $(95 \% \mathrm{Cl}$ $2.2-16.8)$ to deliver an infant with PPHN than women who did not report use of an SSRI during this period of their pregnancy; the absolute risk was low at 6-12 per 1000 live births. Although the authors suggest that the association between SSRIs and PPHN is biologically plausible (SSRIs inhibit the synthesis of nitric oxide and increase levels of serotonin in the lung promoting proliferation of smooth muscle cells which is characteristic of
PPHN), the analysis was based on data from 25 women. Exposure to SSRIs before the 20th week of gestation or exposure to non-SSRI antidepressants at any time during pregnancy was not associated with an increase in the risk of PPHN in this study. It has been suggested that PPHN represents the severe end of a spectrum, with mild neonatal withdrawal symptoms at the other end (Chambers et al, 2006). This seems a plausible explanation for a range of outcomes that have been associated with SSRI exposure in utero:

- commonly, small reductions in gestational age (Simon et al, 2002; Kallen, 2004; Malm et al, 2005; Wogelius et al, 2005; Wen et al, 2006) and SSRI withdrawal symptoms (Zeskind \& Stephens, 2005; LevinsonCastiel et al, 2006)

- less commonly lower Apgar scores in the absence of prematurity (Simon et al, 2002), increased perinatal complications (Diav-Citrin et al, 2005) and increased admissions to intensive care (Malm et al, 2005)

- rarely, PPHN (Chambers et al, 2006).

A further case-control study of 5357 infants with selected major birth defects and 3366 controls has been published (Alwan et al, 2005). Women who were prescribed an SSRI during the first trimester were 3 times more likely (95\% Cl 1.4-6.1) to deliver an infant with omphalocele $(n=161)$ and 1.8 times more likely $(95 \% \mathrm{Cl}$ $1.0-3.2)$ to deliver an infant with craniosyntosis $(n=372)$. This study is currently available in abstract form only. It is unclear how many birth defects were included and how many statistical tests were conducted, therefore these findings should be treated with caution.

\section{Clinical implications}

There is some evidence to suggest an association between first-trimester exposure to SSRIs and a small increase in the rate of congenital malformations, particularly cardiac malformations with paroxetine. Most of the suggested associations have not been replicated and it is difficult to interpret those identified through casecontrol studies as most reports are only available in abstract form. There is, however, increasing evidence to suggest an association between SSRI exposure during pregnancy and neonatal withdrawal symptoms, and additionally, a small reduction in gestational age. It is important to be aware of these findings when considering the use of SSRIs in women of child-bearing age. Until further conclusive evidence is available regarding the use of paroxetine in pregnancy other SSRIs should be considered first. If a woman conceives while taking paroxetine this is not necessarily an indication to discontinue therapy or switch to an alternative antidepressant. The risks and benefits should be evaluated and a joint decision made between prescriber and patient regarding treatment. It might be possible to avoid the effects of exposure to SSRIs in late pregnancy by tapering antidepressants during the third trimester; however consideration should be given to the high risk of relapse on stopping therapy. 
medicines information

\section{References}

ALWAN, S., REEFHUIS, J., RASMUSSEN, DIAV-CITRIN, O., SHECHTMAN, S., S., et al (2005) Maternal use of selective WEINBAUM, D., et al (2005) Paroxetine serotonin reuptake inhibitors and risk for birth defect. Clinical and Molecular Teratology, 731, 291.

CHAMBERS, C. D., HERNANDEZ-DIAZ, S., VAN MARTER, L. J., et al (2006) Selective serotonin reuptake inhibitors and risk of persistent pulmonary hypertension of the newborn. New England Journal of Medicine, 354 579-588.

COHEN, L. S., SICHEL, D. A., ROBERTSON, L. M., et al (1995) Postpartum prophylaxis for women with bipolar disorder. American Journal of Psychiatry, 152, 1641-1644.

COHEN, L. S., ALTSHULER, L. L., HARLOW, B. L., et al (2006) Relapse of major depression during pregnancy in women who maintain or discontinue antidepressant treatment. JAMA, 295 499-507.
LEVINSON-CASTIEL, R., MERLOB, P.,

KALLEN, B. (2004) Neonate characteristics after maternal use of antidepressants in late pregnancy. Archives of Pediatric Adolescent Medicine, 158, 312-316. LINDER, N., et al (2006) Neonatal abstinence syndrome after in-utero exposure to selective serotonin reuptake inhibitors in term infants. Archives of Pediatric Adolescent Medicine, 160, 173-176.

MALM, H., KLAUKKA, T., NEUVONEN, P. J. (2005) Risks associated with selective serotonin reuptake inhibitors in pregnancy. Obstetrics and Gynecology, 106, 1289-1296. NATIONAL INSTITUTE FOR CLINICAL EXCELLENCE (2004) Depression: Management of Depression in Primary and Secondary Care. NICE.

NELSON, K. \& HOLMES, L. B. (1989) Malformations due to presumed spontaneous mutations in newborn infants. New England Journal of Medicine, 320, 19-23.

SANZ, E. J., DE-LAS-CUEVAS, C., KIURU, A., et al (2005) Selective serotonin reuptake inhibitors in pregnant women and neonatal withdrawal syndrome: a database analysis. Lancet, 365, 482-487.

SIMON, G. E., CUNNINGHAM, M. L., DAVIS, R. L. (2002) Outcomes of prenatal antidepressant exposure. American Journal of Psychiatry, 159, 2055-2061.

WEN, S.W., YAND, Q., GARNER, P., et al (2006) Selective serotonin reuptake inhibitors and adverse pregnancy outcomes. American Journal of Obstetrics and Gynecology, 194, 961966.

WOGELIUS, P., NORGAARD, M., MUNK, E. M., et al (2005) Maternal use of selective serotonin reuptake inhibitors and risk of adverse pregnancy outcome. Pharmacoepidemiology and Drug Safety, 14, S72.

WORLD HEALTH ORGANIZATION (2001) Fact Sheet: The World Health Report. Mental and Neurological Disorders. WHO.

ZESKIND, P. S. \& STEPHENS, L. E. (2005) Maternal selective serotonin reuptake inhibitor use during pregnancy and newborn neurobehaviours. Paediatrics, $113,368-375$

Allison Donnelly Clinical Pharmacist, Oxleas NHS FoundationTrust,

*Carol Paton Chief Pharmacist, Oxleas NHS FoundationTrust, Pinewood House, Pinewood Place, Dartford, Kent DA2 7WG, email: Carol.Paton@oxleas.nhs.uk 